\title{
Medial Temporal Lobe Damage Causes Deficits in Episodic Memory and Episodic Future Thinking Not Attributable to Deficits in Narrative Construction
}

\author{
Elizabeth Race, ${ }^{1,2}$ Margaret M. Keane, ${ }^{1,2,3}$ and Mieke Verfaellie ${ }^{1,2}$ \\ ${ }^{1}$ VA Boston Healthcare System and ${ }^{2}$ Boston University School of Medicine, Boston, Massachusetts 02130, and ${ }^{3}$ Department of Psychology, Wellesley \\ College, Wellesley, Massachusetts 02481
}

The medial temporal lobe (MTL) makes critical contributions to episodic memory, but its contributions to episodic future thinking remain a matter of debate. By one view, imagining future events relies on MTL mechanisms that also support memory for past events. Alternatively, it has recently been suggested that future thinking is independent of MTL-mediated processes and can be supported by regions outside the MTL. The current study investigated the nature and necessity of MTL involvement in imagining the future and tested the novel hypothesis that the MTL contributes to future thinking by supporting online binding processes related to narrative construction. Human amnesic patients with well characterized MTL damage and healthy controls constructed narratives about (1) future events, (2) past events, and (3) visually presented pictures. While all three tasks place similar demands on narrative construction, only the past and future conditions require memory/future thinking to mentally generate relevant narrative information. Patients produced impoverished descriptions of both past and future events but were unimpaired at producing detailed picture narratives. In addition, futurethinking performance positively correlated with episodic memory performance but did not correlate with picture narrative performance. Finally, future-thinking impairments were present when MTL lesions were restricted to the hippocampus and did not depend on the presence of neural damage outside the MTL. These results indicate that the ability to generate and maintain a detailed narrative is preserved in amnesia and suggest that a common MTL mechanism supports both episodic memory and episodic future thinking.

\section{Introduction}

While it is well established that the medial temporal lobe (MTL) plays a critical role in remembering the past, recent data suggest that the MTL, and particularly the hippocampus, also supports imagining the future. MTL activity increases when subjects envision future events (Okuda et al., 2003; Addis et al., 2007; Hassabis et al., 2007a; Schacter and Addis, 2007), and amnesic patients with MTL damage and severe autobiographical (episodic) memory loss demonstrate striking impairments in the ability to imagine the future (Tulving, 1985; Klein et al., 2002; Hassabis et al., 2007b; Andelman et al., 2010). Together, these results have been taken as evidence that future thinking relies on MTL mechanisms that also support episodic memory (Eichenbaum and Fortin, 2009; Hassabis and Maguire, 2009; Schacter and Addis, 2009; Buckner, 2010).

A recent study has challenged this view by demonstrating that amnesic patients with MTL damage can imagine future events as

\footnotetext{
Received March 4, 2011; revised May 3, 2011; accepted May 23, 2011.

Author contributions: E.R., M.M.K., and M.V. designed research; E.R. performed research; E.R. analyzed data; E.R., M.M.K., and M.V. wrote the paper.

This research was supported by the Office of Research and Development, Medical Research Service, Department of Veterans Affairs.

The authors declare no competing financial interests.

Correspondence should be addressed to Elizabeth Race, Memory Disorders Research Center, VA Boston Healthcare System, 150 South Huntington Avenue (151-A), Boston, MA 02130. E-mail: race@bu.edu.

DOI:10.1523/JNEUROSCI.1145-11.2011

Copyright $\odot 2011$ the authors $\quad 0270-6474 / 11 / 3110262-08 \$ 15.00 / 0$
}

well as controls (Squire et al., 2010). The authors concluded that the MTL is not necessary for future thinking, and suggested that prior reports of impaired future thinking in amnesia reflect neural damage outside the MTL. Because the extent of neurological damage in prior amnesia studies was either not quantified or included extra-MTL regions, a critical outstanding question is whether future-thinking impairments result from damage specific to the MTL.

Differences across studies in factors related to patient performance may provide additional insight into this debate. In contrast to prior amnesia studies, the patients in the Squire et al. (2010) study did not demonstrate pervasive autobiographical memory loss, suggesting that the extent of future-thinking and memory impairments in amnesia may be related. If a positive correlation exists between the magnitude of memory and futurethinking impairments in amnesia, this would support the hypothesis that a common MTL mechanism underlies both functions. Additionally, the amnesic patients in the Squire et al. (2010) study produced more repetitions during their event descriptions than controls, suggesting that MTL damage may impair the online integration of narrative information in working memory (Hannula et al., 2006; Olson et al., 2006; Luck et al., 2010). An important question is whether impaired future thinking in amnesia reflects deficits in MTL mechanisms supporting narrative construction.

The current study investigates the nature and necessity of MTL involvement in future thinking by testing the ability of am- 
Table 1. Demographic, neuropsychological, and neurological characteristics of amnesic patients

\begin{tabular}{lllllllllll}
\hline & & & & \multirow{2}{*}{ WAIS, III: } & \multicolumn{5}{c}{ WMS, III } & \\
\cline { 6 - 8 } Patient & Etiology & Age & Edu & VIQ & & GM & VD & AD & WM & Vol Loss \\
\hline P01 & Encephalitis & 55 & 14 & 92 & 45 & 56 & 55 & 85 & $73 \%$ \\
P02 & Encephalitis & 66 & 12 & 106 & 69 & 68 & 77 & 111 & $66 \%$ \\
P03 & Anoxia & 60 & 12 & 83 & 52 & 56 & 55 & 91 & N/A \\
P04 & Anoxia & 46 & 16 & 86 & 49 & 53 & 52 & 93 & $63 \%$ \\
P05 & Anoxia & 54 & 14 & 111 & 59 & 72 & 52 & 96 & $27 \%$ \\
P06 & Encephalitis & 82 & 18 & 135 & 45 & 53 & 58 & 141 & N/A \\
P07 & Anoxia & 58 & 17 & 134 & 70 & 75 & 67 & 126 & N/A \\
P08 & Anoxia & 60 & 16 & 110 & 62 & 68 & 61 & 92 & N/A \\
\hline
\end{tabular}

Age, Age in years; Edu, education in years; WAIS, III, Wechsler Adult Intelligence Scale, III; VIQ, verbal IQ; WMS, III, Wechsler Memory Scale, III; GM, general memory; VD, visual delayed; $A D$, auditory delayed; WM, working memory; Vol Loss, hippocampal volume loss.

nesic patients with well characterized MTL lesions to construct narratives about (1) recent/remote past events, (2) near/distant future events, and (3) visually presented pictures. While these conditions place similar demands on narrative construction, only the past and future conditions require memory/future thinking to mentally generate relevant narrative information. If the MTL is necessary for imagining the future, patients should demonstrate impaired performance in the future condition. If a common MTL mechanism supports both memory and future thinking, the magnitude of patients' impairment in the future condition should positively correlate with the magnitude of their impairment in the past condition. Finally, if future thinking specifically depends on MTL mechanisms related to narrative construction, patients should demonstrate parallel impairments across all three conditions.

\section{Materials and Methods}

Participants. Eight amnesic patients (three female) with MTL lesions participated in the study (Table 1). The neuropsychological profiles of all patients indicate impairments isolated to the domain of memory. Five of the patients had an etiology of anoxia, and the remaining three patients had an etiology of herpes encephalitis. To assess the extent of patients' neural damage, structural magnetic resonance imaging (MRI) scans were collected for two of the encephalitic patients and for two of the anoxic patients (MRI could not be obtained for the remaining patients because of medical contraindications, but MTL pathology can be inferred on the basis of etiology and neuropsychological profile). Information about the acquisition and analysis of MRI scans has been previously reported (Kan et al., 2007). For lesion volumetric analysis of medial temporal lobe regions, the hippocampus and amygdala were individually segmented according to established parameters (Seidman et al., 2002). The parahippocampal gyrus was defined anteriorly by the isthmus of the temporal and frontal lobes, medially by the collateral fissure, laterally by the hippocampal fissure, and posteriorly by the anterior limit of the calcarine fissure. Regional brain volumes were determined by multiplying the number of voxels within a parcellation unit on a given coronal slice by the voxel volume and summing across all slices in which each unit appeared.

Quantitative analysis compared patients' regional brain volumes (corrected for intracranial volume) to volumes from eight age- and gendermatched control subjects. One of the anoxic patients (P05) had damage limited to the hippocampus and two of the encephalitic patients (P01 and $\mathrm{P} 02$ ) and one of the anoxic patients (P04) had damage to the hippocampus and to the surrounding parahippocampal gyrus (volume reductions $>2$ SDs from the control mean). P05 had unilateral reduction in right hippocampal volume of $27 \%$, and $\mathrm{P} 01, \mathrm{P} 02$, and $\mathrm{P} 04$ had bilateral reductions in hippocampal volume of $73 \%, 66 \%$, and $63 \%$, respectively. Measurements of frontal, parietal, occipital, and lateral temporal cortex were also made to assess the possibility of additional damage outside the MTL. The hippocampus was the single area of overlap across all participants and no common volume reductions were found outside the MTL. The only extra-MTL volume reductions were observed in two individual subjects and were restricted to the right lateral temporal lobe for P02 and to the left lateral temporal lobe for P04.

Twelve healthy controls also participated (six female). The control group was matched to the patient group in terms of mean age $(60 \pm 12.2$ years), mean education ( $14 \pm 2.0$ years), and mean verbal IQ (105 \pm 15.7). All subjects provided informed consent in accordance with the procedures of the Institutional Review Boards at Boston University and the VA Boston Healthcare System.

Materials and procedure. For the past and future event tasks, questionnaires were formed requiring participants to (1) recollect 10 specific personal events from the past (e.g., graduation ceremony) and (2) imagine 10 specific personal events in the future (e.g., winning the lottery). Half of the past and future events occurred close in time to the present (e.g., the recent past, "one year ago," and the near future, "next year"), and half of the past and future events occurred farther in time from the present (e.g., the remote past, "when you were a teenager," and the distant future, "in twenty years"). Twenty years was selected as the timeframe for distant future events as a compromise between matching the temporal distance of distant past and future events, and specifying a future time point that would fall within a plausible lifetime period for the participants given their current age.

For each event, a short description was read aloud by the experimenter and participants were instructed to describe the event in as much detail as possible (e.g., "Imagine catching your grandchild getting into trouble twenty years from now. Describe in as much detail as you can what this event will be like. Describe where and when you catch your grandchild getting into trouble, who is there, how you feel, and what you are thinking"). Participants were required to describe specific, preexperimentally determined events to minimize descriptions of repeated events or frequently retrieved event information. The specificity of the event cues is especially important given that the preferred level of processing in autobiographical memory is at a more general level (Conway, 2001), and patients with temporal lobe lesions have been shown to rely on generic autobiographical knowledge to fill gaps in their memories for past events (Thaiss and Petrides, 2008).

Within the allotted $3 \mathrm{~min}$, participants continued with their descriptions without interference from the examiner until they came to a natural ending point. The examiner then provided a single, standardized probe to elicit additional details (e.g., "Can you tell me any more about where and when the event is taking place, who is there, how you feel, and what you are thinking?"), and participants were given another 3 min to elaborate without interference from the examiner. This standardized probing protocol was chosen to minimize variability in levels of retrieval support provided by the examiner and to control for variable interactional contexts and social negotiations between the experimenter and participants. This standardization across participants is important given that unconstrained probing protocols and repeated questioning by the experimenter have recently been shown to introduce uncontrolled interactional contexts and social negotiations that can affect subjects' responses to an open-ended question (McKinlay et al., 2010). In addition, it is known that amnesic patients use personal and communal knowledge derived from interactions between experimenter and subject differently than do controls during collaborative discourse (Duff et al., 2008).

For the picture narrative condition, participants were shown five detailed drawings of scenes, one at a time, that depicted characters engaged in various activities (e.g., a park scene) (see Fig. 5A). For each picture, participants were instructed to imagine that the picture was a scene taken from a movie and to tell a story about what was going on in the scene (e.g., "Imagine what the movie is about and tell a story about what's going on in the scene. What is happening in the scene and how did this scene come about? What are the characters in the scene doing, feeling, thinking and why?"). Participants were instructed to tell a story about the scene rather than to report only what was literally depicted in the picture to more closely match the narrative construction demands in the picture condition to those of the future/past conditions. The picture remained in front of the participants for the duration of the task and participants were given 
up to $3 \mathrm{~min}$ for their narrative. Within these $3 \mathrm{~min}$, participants were allowed to continue uninterrupted until they came to a natural ending point. Critically, the use of visually rich pictures to cue participants' narratives in the picture condition eliminated the need to mentally generate descriptive elements (e.g., relevant and coherent scene/event information) while retaining cognitive demands related to the narrative nature of the task (e.g., verbal production and working memory demands). This contrasts with the use of auditory/verbal cues in the future/ past conditions that do not provide relevant narrative elements to participants. While this manipulation necessitated using different modalities of cues across conditions, it enabled the critical comparison of narrative performance when relevant descriptive elements must be mentally generated (future/past conditions) to narrative performance when descriptive elements are experimentally provided (picture condition).

Participants participated in three testing sessions, one picture narrative session and two event narrative sessions, with the order of sessions counterbalanced across participants. Within each of the event narrative sessions, participants described both personal events and public events occurring in each of the past and future time periods. (Data from public events are not presented in the current paper.) Trials were blocked by condition (personal/public events), but presentation order of conditions was counterbalanced across sessions and participants. Similarly, within the personal event condition, trials were blocked by temporal direction (future, past) and temporal distance (remote/distant, recent/near). Future events were always presented before past events to ensure that performance on the future-thinking trials was not affected by performance differences on the memory trials, but the presentation order of trials from each temporal distance was counterbalanced across sessions and participants. Testing sessions were digitally recorded for subsequent transcription and scoring.

Scoring. Narratives were scored using an adapted autobiographical interview scoring procedure (Levine et al., 2002). Each narrative was first segmented into distinct details and then each detail was categorized as an episodic detail, a semantic detail, an external detail, a repetition, or a metacomment about the task. Episodic details comprised happenings and individuals present and were further categorized as event, place, time, perceptual, and thought/emotion details. Semantic details comprised general knowledge and facts, ongoing events, and extended states of being, and were further categorized as general semantic, semanticautobiographical, semantic-time, and semantic-place details. External details comprised details from incidents external to the main event being described. The same scoring criteria were used for the future, past, and picture conditions, with the exception that an additional episodic detail category (object-location details) was included for the picture narrative task in addition to the event location (place) category, as it was directly relevant to the description of scenes. For the picture narrative task, inferences about the picture were scored as external details only if they were off topic and external to the main event being described.

For each future/past narrative, the number of details in each category was counted for each subject and averaged across the five events in each time period (remote past, recent past, near future, distant future). Interrater reliability of scoring was established on the basis of 40 event narratives scored by two raters (an equal number of future/past and patient/ control narratives were scored). Following methods used in prior studies (Levine et al., 2002; Hassabis et al., 2007b), the primary scorer was not blind to subject status, but the second trained scorer was blind to subject status. Intraclass correlation analysis indicated high agreement across scorers for future events (Cronbach's $\alpha=0.99$ for total details, $\alpha=0.99$ for total semantic details, $\alpha=0.98$ for total episodic details; range $=$ 0.83-0.99 for each of the subcategories of episodic details) as well as high agreement across scorers for past events (Cronbach's $\alpha=0.99$ for total details, $\alpha=0.95$ for total semantic details, $\alpha=0.98$ for total episodic details; range $=0.70-0.99$ for each of the subcategories of episodic details).

For each picture narrative, the number of details in each category was counted and the totals were averaged across the five picture trials for each subject. Interrater reliability of scoring was established on the basis of 10 events scored by two raters (an equal number of patient/control narratives were scored). Intraclass correlation again indicated high agreement
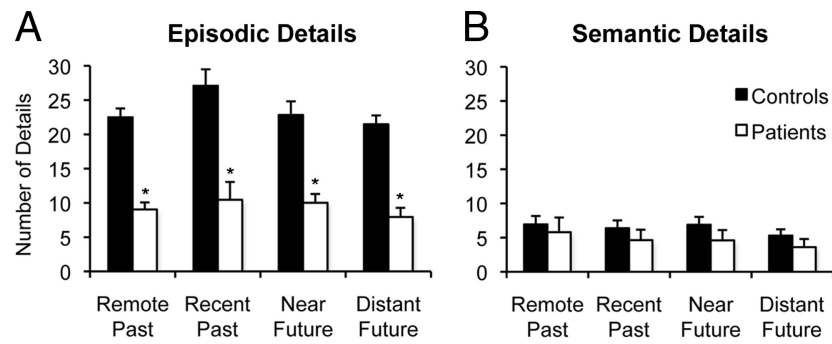

Figure 1. Memory and future-thinking performance. Mean number of episodic details $(\boldsymbol{A})$ and semantic details $(\boldsymbol{B})$ generated by MTL patients and controls for remote past, recent past, near future, and far future events. Error bars indicate SEM. ${ }^{*} p<0.001$ compared to controls.

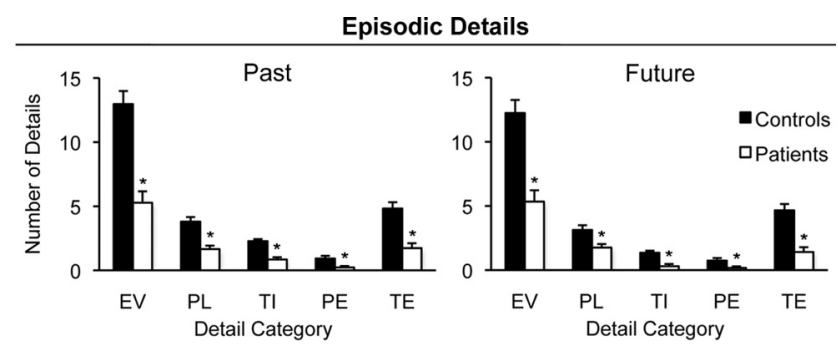

Figure 2. Mean number of episodic details generated by patients and controls in each episodic detail category for past and future events (averaged across recent/remote and near/ distant temporal distances, respectively). Detail categories are defined as follows: $\mathrm{EV}$, event; $\mathrm{PL}$, place; TI, time; PE, perceptual; TE, thought/emotion. Error bars indicate SEM. ${ }^{*} p<0.05$ compared to controls.

across scorers (Cronbach's $\alpha=0.99$ for total details, $\alpha=0.78$ for total semantic details, $\alpha=0.99$ for total episodic details; range $0.72-0.99$ for each of the subcategories of episodic details).

\section{Results}

Figure 1 presents performance on the future and past event narratives. Data for episodic and semantic details were separately analyzed by three-way mixed-factorial ANOVAs with factors of group (patient vs control), temporal direction (past vs future), and temporal distance (remote/distant vs recent/near). Patients produced fewer episodic details than controls (Fig. $1 A)\left(F_{(1,18)}=\right.$ $44.28, p<0.001)$, but both groups produced a similar number of semantic details (Fig. $1 B)\left(F_{(1,18)}=0.98, p=0.33\right)$. In terms of episodic details, participants produced a greater number of details for past than future events (main effect of temporal direction, $\left.F_{(1,18)}=4.81, p=0.04\right)$ and for temporally close than temporally distant events (main effect of temporal distance, $\left.F_{(1,18)}=5.33, p=0.03\right)$. However, the extent of patients' impairment in episodic detail generation did not differ according to temporal direction (group $\times$ direction interaction, $F_{(1,18)}=1.47$ $p=0.24$ ) or temporal distance (group $\times$ distance interaction, $\left.F_{(1,18)}=0.37, p=0.55\right)$, and follow-up pairwise analyses indicated that patients produced fewer episodic details than controls within each time period ( $t$ values $>4.59$, $p$ values $<0.001$ ). Given that patients' impairment in episodic detail generation did not differ according to the temporal distance of past and future events, subsequent analyses comparing memory and futurethinking performance across groups collapse over temporal distance.

Figure 2 presents the number of details produced within each of the episodic detail categories (event, place, time, perceptual, and thought/emotion) for past and future events. Data were submitted to a $2 \times 2 \times 5$ mixed-factorial ANOVA with factors of group, temporal direction, and detail category. There was a main 
Cue: Imagine catching your grandchild getting into trouble twenty years from now.

Patient: Twenty years from now, I'll be in my seventies. I catch my grandchild drinking and I explain the trouble I got into as a teenager drinking, what drinking can lead to in the future, prolonged use of it, as well as the short-term effects, and hopefully get them on the right path. I would imagine I would be visiting and I would be thinking I wish I was that young again and stupid. It's kind of hard to imagine stuff.

Control: This grandchild has come to visit me and I'm living in a senior center by that time with my own apartment. The child has come on her own, flown in. What the child is doing is doping. That's still an issue. I know it because I can smell it. I approach the child in the afternoon, it's been going on the night before after I went to bed and she's in a single room, it's not a big complex that l'm in. The next morning I have a conversation with her. I don't accuse, I just ask how long. I assume she knows that I know. I have a discussion in that way. I ask her what's going on in her schools. I'll ask if her family knows that she's using. When she goes out the next morning to pick up groceries I discover the butts and some ashes. So the conversation is a hard conversation. By that time she's in her twenties so we have a straightforward discussion about how this is affecting her life. I'll ask her not to do drugs in my house. I'm troubled and l'm angry a little bit because she lies at first. It's just going to be me and that child. For me the relationship for that child is much more important, I'm not wanting to get her into difficulty. I don't know in twenty years hence that it'll be a problem.

Figure 3. Representative sample of future event narratives generated by amnesic patient P05 (top) and a control subject (bottom) when instructed to imagine catching a grandchild getting into trouble 20 years from now.

effect of detail category $\left(F_{(4,72)}=117.58, p<0.001\right)$, and follow-up pairwise analyses indicated that the event category yielded more details than every other category ( $t$ values $>$ 8.10 , $p$ values $<0.001)$. Although the extent of patients' impairment differed according to category (group $\times$ category interaction, $F_{(4,18)}=18.82, p<0.001$ ), follow-up analyses indicated that patients produced fewer episodic details than controls within each category both for past events ( $t$ values $>2.55$, $p$ values $<$ $0.05)$ and future events ( $t$ values $>2.64$, $p$ values $<0.05$ ).

Both controls and patients produced very few repetitions during their past event narratives [mean $(\mathrm{SD})=1.08(0.49)$ and 0.13 (0.16), respectively] and their future event narratives [mean $(\mathrm{SD})=0.63(0.41)$ and $0.10(0.13)$, respectively]. When the total number of words produced during the future/past narratives was entered into a three-way mixed-factorial ANOVA with factors of group, temporal direction, and temporal distance, a significant main effect of group indicated that patients produced fewer total words than controls $\left(F_{(1,18)}=4.50, p=0.05\right)$. However, this difference in word count between groups did not differ across future and past time periods (group $\times$ temporal direction interaction, $\left.F_{(1,18)}=0.72, p=0.41\right)$, nor across temporal distance (group $\times$ temporal distance interaction, $F_{(1,18)}=0.93, p=0.35$ ).

To examine the degree to which patients' memory and future-thinking impairments related to hippocampal or extrahippocampal MTL damage, data from the patient with confirmed hippocampal-only damage (P05) were considered separately. Patient P05's performance mirrored that of the patient group as a whole: Patient P05 produced significantly fewer episodic details compared to controls for both past events (11.6 details) and future events (9.0 details) ( $t$ values $>$ 7.85 , $p$ values $<0.001 ; z$ scores $>2$ SDs from the control means). This pattern of impairment was also present across all temporal distances (remote past, recent past, near future, far future: $t$ values $>5.90, p$ values $<0.001)$. Because impairments were observed in a patient without extensive MTL lesions, these results suggest that damage specific to the hippocampus may be sufficient to impair event construction during memory and future thinking. Example future event narratives from P05 and a control subject are reported in Figure 3.

To examine whether experimenter cuing affected the magnitude of participants' memory and future-thinking impairments, the number of episodic details produced before the cuing probe (pre-probe) was compared to the number of episodic details produced in total (pre-probe + post-probe) (Fig. 4). Data were submitted to a three-way mixed-factorial ANOVA with factors of group, temporal direction, and probe (pre-probe details vs total details). A significant group $\times$ probe interaction $\left(F_{(1,18)}=51.45, p<0.001\right)$ reflected that pa-

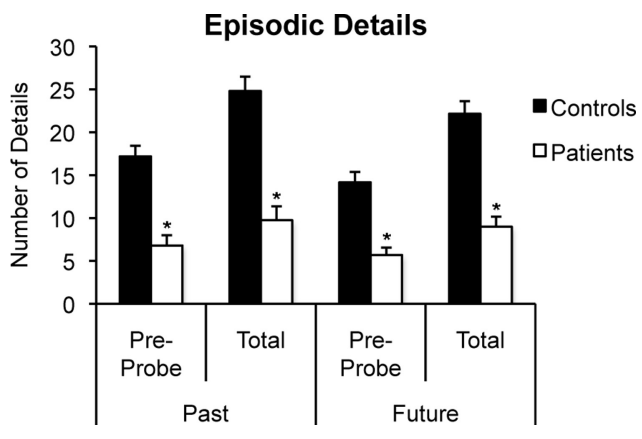

Figure 4. Mean number of episodic details generated by patients and controls before the cuing probe (pre-probe) and in total (pre-probe + post-probe) for past and future events. Error bars indicate SEM. * $p<0.001$ compared to controls.

tients' impairment in episodic detail generation was smaller when performance was measured before the cuing probe. However, follow-up analysis revealed that patients' deficit in episodic detail generation was significant even before the cuing probe for both past events $\left(F_{(1,18)}=31.30, p<0.001\right)$ and future events $\left(F_{(1,18)}=24.52, p<0.001\right)$.

Next, performance on the picture narrative task was analyzed to measure patients' ability to construct an extended narrative that requires integrating information in working memory but does not require the mental generation of descriptive elements (e.g., relevant and coherent scene/event information). Picture narrative data were entered into a two-way mixed-factorial ANOVA with factors of group (patient vs control) and detail type (episodic vs semantic). In contrast to the distinct patterns of performance across patients and controls in the past and future narratives, both groups produced a similar number of details for the picture narratives (main effect of group, $F_{(1,18)}=0.08, p=$ 0.78 ). The majority of details produced during picture narratives were episodic in nature (main effect of detail type, $F_{(1,18)}=$ $105.80, p<0.001)$. Controls produced an average of $22.1(\mathrm{SD}=$ 6.9) episodic details and $1.3(\mathrm{SD}=1.6)$ semantic details per narrative, and patients produced an average of $22.0(\mathrm{SD}=11.4)$ episodic details and $0.2(\mathrm{SD}=0.2)$ semantic details per narrative. Example picture narratives from a patient and a control subject are reported in Figure $5 B$.

Figure 6 displays the mean number of episodic details produced by patients and controls within each episodic detail category during picture narrative performance. When the data were submitted to ANOVA with factors of group and episodic detail category, there was a main effect of episodic detail category $\left(F_{(5,90)}=35.23, p<0.001\right)$ but no group $\times$ category interaction $\left(F_{(5,18)}=1.61, p=0.21\right)$. In addition, patients did not produce a 
A

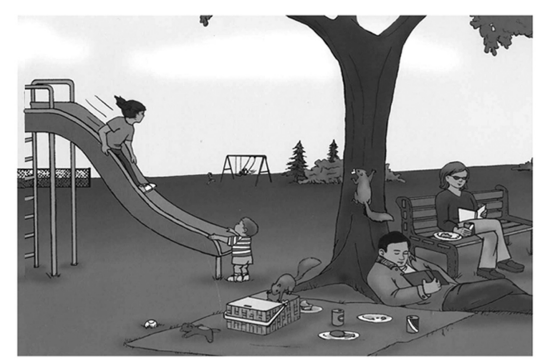

B

Cue: Imagine the picture is a scene taken from a movie and tell a story about what's going on in the scene.

Patient: It's a movie about a family who are at the park, two children, a boy and a girl. The boy looks younger than the girl. The girl is coming down the slide. Also children a ways away, two, one running to a swing and another running away from it. The squirrels are having a field day. One is going up a tree with something in its mouth that it took from the basket. The other one is going into the basket. Dad or the man is sleeping, ignoring everything. The mother looks like she was eating, she has a can of something in her hand and she's reading a book and totally oblivious to the children. The little boy also has a teddy bear that he brought that's on the blanket. Looks like they were eating a chicken leg and muffin. Two cans of soda on the blanket and a baseball nearby.

Control: The movie could be a day at the park. You could see the mother sitting on the bench, reading a book. The father's under a tree. One squirrel climbing up the tree, another squirrel into the lunch basket. Two people, a young lady sliding down a slide and a little boy at the end of it. Beyond them is a swing. There are trees, a red blanket, food in the basket, couple cans of soda, plate. Lady reading the book has a plate on the bench. Everybody seems to be having a good time. The father's sleeping and the mother's reading, kids are sliding. And they live happily ever after.

Figure 5. Representative sample of a picture used for the picture narrative condition $(\boldsymbol{A})$ and representative sample of picture narratives generated by an amnesic patient (top) and a control subject (bottom) when instructed to describe a story about what's going on in the scene (B).

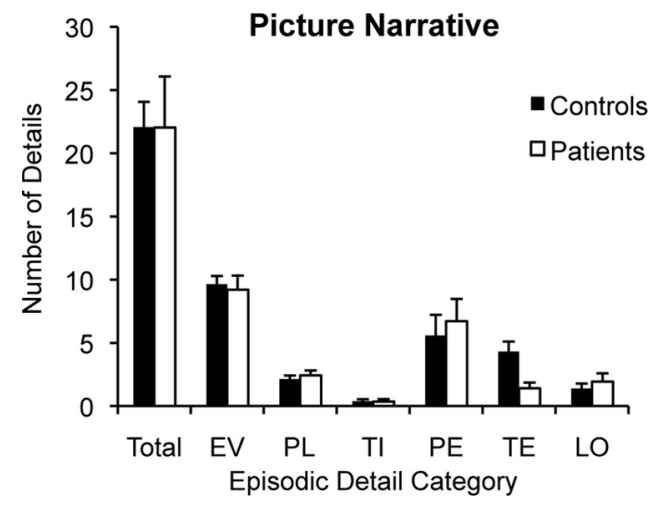

Figure 6. Picture narrative performance. Mean number of episodic details generated by patients and controls during picture narratives. Detail categories are defined as follows: EV, event; PL, place; II, time; PE, perceptual; TE, thought/emotion; L0, object-location. Error bars indicate SEM.

greater number of repetitions than controls during the picture narrative task $\left(t_{(18)}=1.02, p=0.32\right)$, with both patients and controls producing fewer than two repetitions on average per narrative. Examination of the distribution of patients' picture narrative scores revealed that one patient had an episodic detail score that was greater than 2 SDs above the patient average (P01). However, even after excluding this participant, patients and controls still produced a similar number of episodic details for picture narratives $(p=0.89)$, and performance did not differ between groups across detail categories $(p=0.14)$. Patients' preserved ability to construct a narrative when relevant scene and event details are provided in a picture argues against general nar-
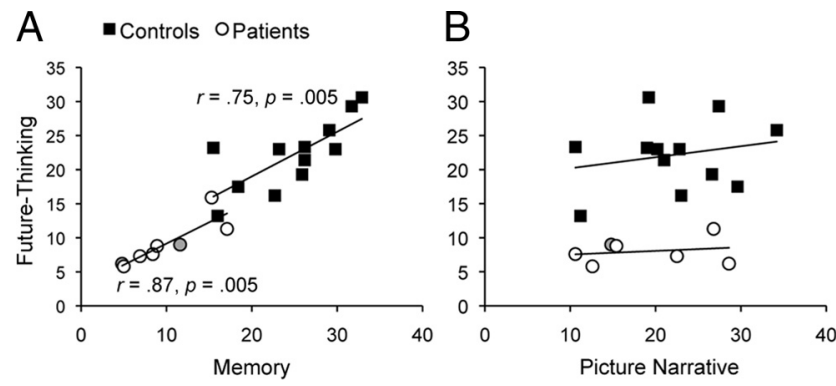

Figure 7. Scatter plots and regression lines showing significant correlations between future-thinking performance and memory performance (number of episodic details generated for future events vs number of episodic details generated for past events) for both controls and patients $(\boldsymbol{A})$ and nonsignificant correlations between future-thinking performance and picture narrative performance (number of episodic details generated for future events vs number of episodic details generated for picture narratives) for both controls and patients (B). Amnesic patients are represented by circles and controls are represented by squares. The data point representing the patient whose MTL lesion is restricted to the hippocampus (P05) is indicated by a light gray fill in both panels. $\boldsymbol{B}$ excludes the patient with outlier performance on the picture narrative task (P01).

rative construction deficits as the mechanism underlying patients' deficit in imagining the future.

To examine the relationship between memory, future thinking, and picture narrative performance, correlations between participants' performance across tasks were computed. Critically, future-thinking performance (number of episodic details generated for future events) positively correlated with performance on the memory task (number of episodic details generated for past events) in both controls $(r=0.75, p=0.005)$ and patients $(r=$ $0.87, p=0.005$ ) (Fig. $7 A$ ). A strong positive correlation was also found when comparing the magnitude of patients' impairment in memory and future thinking when performance was calculated as $z$ scores $(r=0.75, p=0.03)$. These results support the hypothesis that a common MTL mechanism is critical for both episodic memory and episodic future thinking. In contrast, futurethinking performance did not correlate with performance on the picture narrative task (number of episodic details generated for picture narratives) in either controls $(r=0.22, p=0.49)$ or patients $(r=0.20, p=0.66$; excluding outlier P01) (Fig. $7 B$ ), providing additional evidence that narrative construction ability is not a significant predictor of memory and future-thinking performance.

To further examine performance differences across tasks, hierarchical multiple regression was performed with futurethinking performance entered as a dependent variable and picture narrative performance, memory performance, and group (patients vs controls) entered as predictors. While picture narrative performance was not a significant predictor of futurethinking performance $\left(R^{2}=0.05, F_{(1,18)}=0.89, p=0.35\right)$, memory performance significantly predicted future-thinking performance when added to the second step of the model $\left(R^{2}\right.$ change $\left.=0.82, F_{(1,17)}=103.26, p<0.001\right)$. Further, adding group to the third step did not improve the model's capacity to account for variance in future-thinking performance $\left(R^{2}\right.$ change $\left.=0.02, F_{(1,16)}=2.70, p=0.12\right)$, indicating that no group-related factors beyond those accounting for variance in memory performance account for variance in future-thinking performance.

\section{Discussion}

The present study provides novel evidence that the MTL plays a critical role in both reexperiencing the past and preexperiencing 
the future. Amnesic patients with well characterized MTL damage were impaired at constructing detailed narratives about personal events in four different time periods (remote past, recent past, near future, distant future) and future-thinking performance strongly correlated with episodic memory performance. Critically, patients' memory and future-thinking impairments starkly contrasted with their preserved ability to construct narratives based on visually presented pictures and did not depend on a particular etiology of amnesia or reflect neural damage outside the MTL. Together, these results support the hypothesis that a common MTL mechanism supports both episodic memory and episodic future thinking, and argue against deficits in higherorder processes related to narrative construction as the underlying cause of future-thinking impairments in amnesia.

The patterns of impaired and preserved function in amnesia shed light on current debates about MTL function. With regard to autobiographical memory, the observation that MTL damage impairs memory for past events regardless of temporal distance challenges standard models of memory consolidation in which the MTL plays a time-limited role in episodic memory retrieval (Squire and Alvarez, 1995; Bayley et al., 2003; Kirwan et al., 2008) and supports multiple trace models in which retrieval of contextually rich episodic memories always remains dependent on the MTL (Nadel and Moscovitch, 1997; Nadel et al., 2000; Steinvorth et al., 2005). With regard to future thinking, the extensive impairments that occur with MTL damage argue against the hypothesis that future thinking is independent of MTL processes (Squire et al., 2010) and favor prospective theories of MTL function in which the MTL supports prediction and imagination (Hassabis and Maguire, 2009; Schacter and Addis, 2009; Buckner, 2010). In addition, the observation that patients generated fewer episodic details for future events across all detail categories and time periods extends initial reports of impaired future thinking in amnesia (Tulving, 1985; Klein et al., 2002; Hassabis et al., 2007b; Rosenbaum et al., 2009; Andelman et al., 2010) and highlights the importance of MTL contributions to future thinking regardless of temporal distance (cf. Addis and Schacter, 2008).

The current study also presents the first report that futurethinking performance in amnesia strongly correlates with episodic memory performance, providing the strongest evidence to date that a common MTL mechanism supports both episodic memory and episodic future thinking (Schacter and Addis, 2007; Schacter et al., 2007; Hassabis and Maguire, 2009). An open question is whether this common MTL mechanism pertains to the retrieval and recombination of elements from episodic memory (Schacter and Addis, 2009) or the generation of coherent spatial contexts into which relevant details can be bound (Hassabis and Maguire, 2007). While the present study was not designed to distinguish between these possibilities, we addressed an important alternative hypothesis that impaired future thinking in amnesia reflects deficits in MTL processes supporting narrative construction.

Recent findings have indicated that deficits in narrative construction may underlie future thinking impairments in older adults (Gaesser et al., 2011). Critically, all prior reports of impaired future thinking in amnesia have come from studies using narrative tasks, and even in the one study in which amnesic patients imagined future events in as much detail as controls, their event narratives had less continuity (more repetition) (Squire et al., 2010). These results raised the possibility that future-thinking deficits in amnesia reflect an inability to integrate information in working memory during narrative construction. Such online integration of information in working memory provides a tempo- rary modeling space for unfolding event simulations (Baddeley, 2000; Suddendorf and Corballis, 2007), and recent neuroimaging and neuropsychological research suggests that these functions may be at least partially supported by the MTL (Mitchell et al., 2000; Hannula et al., 2006; Olson et al., 2006; Finke et al., 2008; Hannula and Ranganath, 2008; Cashdollar et al., 2009; Piekema et al., 2009; Luck et al., 2010; but see Baddeley et al., 2010; Jeneson et al., 2010). Nonetheless, in the present study, patients' picture narratives contained a similar number of episodic details as controls' and did not contain a greater number of repetitions, indicating that narrative construction does not depend on MTL mechanisms when descriptive elements are readily available (e.g., relevant scene and event details). In addition, patients' performance on the picture narrative task did not correlate with their performance on the future-thinking task, providing further evidence that future-thinking impairments do not simply reflect deficits in narrative construction.

While our results indicate that MTL damage does not disrupt narrative performance when event descriptions can be based on visually presented pictures, it is still possible that the MTL makes important contributions to narrative performance that were not captured by our performance measures. In particular, the current study measured the quantity and quality of individual narrative details (narrative content) but did not examine potential deficits in detail integration (narrative organization) that could impair event construction in amnesia (Rosenbaum et al., 2009). Future studies could assess this possibility using narrative discourse analysis techniques that measure narrative coherence and cohesion (Caspari and Parkinson, 2000).

The severe future-thinking impairments observed in the current study stand in contrast to a recent report of preserved future thinking in amnesia (Squire et al., 2010). Squire et al. (2010) reported that amnesic patients with hippocampal damage were able to imagine events occurring in the near future and suggested that prior reports of impaired future thinking in amnesia reflected pathology in extra-MTL regions involved in remembering autobiographical episodes. However, our volumetric analyses argue against this possibility: no common neural damage was found outside the MTL in our patient group, and the patient with selective hippocampal damage demonstrated impairments as severe as the patients with more widespread cortical damage. Instead, we propose that the divergent results across studies may reflect the fact that future thinking does not depend on the MTL when future events can be constructed from readily available information (e.g., when relevant scene/event information is provided in pictures or can be accessed without the MTL). Indeed, the patients in the study by Squire et al. (2010) did not demonstrate pervasive autobiographical memory loss. By one view (Schacter and Addis, 2009), this preserved mnemonic information could have provided the building blocks for future event simulations. By an alternative view, while performance on the remote memory and future thinking tasks appeared to be spared in the Squire et al. (2010) study, patients' descriptions may not have been as spatially coherent as controls' and may have been more semantic in nature (Maguire and Hassabis, 2011).

An important methodological difference between the current study and the study by Squire et al. (2010) may be particularly relevant to this point. In the current study (and all prior studies of future thinking in amnesia), participants were required to construct specific, preexperimentally determined events (e.g., "winning the lottery"), while in the study by Squire et al. (2010), participants were provided with simple noun cues (e.g., "tree") and were not required to describe events related to the cue. When 
not required to imagine specific events, patients may be able to construct simulations based on well established scripts in semantic memory or generalized memory for routine events that do not place high demands on MTL-mediated processes (see also Maguire et al., 2010; Cooper et al., 2011). Indeed, information that has become overlearned or routinized can be retrieved independently of the MTL (Steinvorth et al., 2005; Leyhe et al., 2010), and the hippocampus is more strongly engaged when participants imagine specific versus general future events and events with low versus high occurrence probability (Addis et al., 2010; Weiler et al., 2010). In line with this possibility, amnesic patients in the current study generated a greater number of details when imagining more frequent versus less frequent events (e.g., birthday celebration vs winning the lottery), and this difference approached significance $(p=0.06)$, although future-thinking impairments in amnesia were significant for both types of specific future events $(p<0.001)$.

In conclusion, the current results indicate that the MTL is critical for constructing event simulations when descriptive elements are not readily available, and suggest that the MTL may be particularly important for constructing future event representations that are both detailed and specific. Future studies can further explore this possibility by examining MTL contributions to semantic future thinking, for example when participants are required to imagine detailed and specific future events occurring in the public domain. Such investigations would also address the possibility that future-thinking deficits in amnesia reflect impairments in self-projection (Buckner and Carroll, 2007; but see Hassabis et al., 2007b). Finally, while the current study focused on MTL contributions to imagining the future, the prospective role of the MTL likely applies more broadly to mental simulation and imagery regardless of temporal specificity (Hassabis and Maguire, 2007; Buckner, 2010). A variety of highly adaptive functions such as planning and problem solving may rely on this remarkable ability to test predictions and mentally explore experiences before they occur (Buckner and Carroll, 2007; Bar, 2009; Eichenbaum and Fortin, 2009; Hassabis and Maguire, 2009; Moulton and Kosslyn, 2009; Schacter and Addis, 2009).

\section{References}

Addis DR, Schacter DL (2008) Constructive episodic simulation: temporal distance and detail of past and future events modulate hippocampal engagement. Hippocampus 18:227-237.

Addis DR, Wong AT, Schacter DL (2007) Remembering the past and imagining the future: common and distinct neural substrates during event construction and elaboration. Neuropsychologia 45:1363-1377.

Addis DR, Cheng T, Roberts RP, Schacter DL (2010) Hippocampal contributions to the episodic simulation of specific and general future events. Hippocampus. Advance online publication. Retrieved November 13, 2010. doi:10.1002/hipo.20870.

Andelman F, Hoofien D, Goldberg I, Aizenstein O, Neufeld MY (2010) Bilateral hippocampal lesion and a selective impairment of the ability for mental time travel. Neurocase 16:426-435.

Baddeley A (2000) The episodic buffer: a new component of working memory? Trends Cogn Sci 4:417-423.

Baddeley A, Allen R, Vargha-Khadem F (2010) Is the hippocampus necessary for visual and verbal binding in working memory? Neuropsychologia 48:1089-1095.

Bar M (2009) The proactive brain: memory for predictions. Philos Trans R Soc Lond B Biol Sci 364:1235-1243.

Bayley PJ, Hopkins RO, Squire LR (2003) Successful recollection of remote autobiographical memories by amnesic patients with medial temporal lobe lesions. Neuron 38:135-144.

Buckner RL (2010) The role of the hippocampus in prediction and imagination. Annu Rev Psychol 61:27-48, C1-C8.

Buckner RL, Carroll DC (2007) Self-projection and the brain. Trends Cogn Sci 11:49-57.
Cashdollar N, Malecki U, Rugg-Gunn FJ, Duncan JS, Lavie N, Duzel E (2009) Hippocampus-dependent and -independent theta-networks of active maintenance. Proc Natl Acad Sci U S A 106:20493-20498.

Caspari I, Parkinson SR (2000) Effects of memory impairment on discourse. J Neurolinguistics 13:15-36.

Conway MA (2001) Sensory-perceptual episodic memory and its context: autobiographical memory. Philos Trans R Soc Lond B Biol Sci 356:1375-1384.

Cooper JM, Vargha-Khadem F, Gadian DG, Maguire EA (2011) The effect of hippocampal damage in children on recalling the past and imagining new experiences. Neuropsychologia 49:1843-1850.

Duff MC, Hengst JA, Tranel D, Cohen NJ (2008) Collaborative discourse facilitates efficient communication and new learning in amnesia. Brain Lang 106:41-54.

Eichenbaum H, Fortin NJ (2009) The neurobiology of memory based predictions. Philos Trans R Soc Lond B Biol Sci 364:1183-1191.

Finke C, Braun M, Ostendorf F, Lehmann TN, Hoffmann KT, Kopp U, Ploner CJ (2008) The human hippocampal formation mediates short-term memory of colour-location associations. Neuropsychologia 46:614-623.

Gaesser B, Sacchetti DC, Addis DR, Schacter DL (2011) Characterizing agerelated changes in remembering the past and imagining the future. Psychol Aging 26:80-84.

Hannula DE, Ranganath C (2008) Medial temporal lobe activity predicts successful relational memory binding. J Neurosci 28:116-124.

Hannula DE, Tranel D, Cohen NJ (2006) The long and the short of it: relational memory impairments in amnesia, even at short lags. J Neurosci 26:8352-8359.

Hassabis D, Maguire EA (2007) Deconstructing episodic memory with construction. Trends Cogn Sci 11:299-306.

Hassabis D, Maguire EA (2009) The construction system of the brain. Philos Trans R Soc Lond B Biol Sci 364:1263-1271.

Hassabis D, Kumaran D, Maguire EA (2007a) Using imagination to understand the neural basis of episodic memory. J Neurosci 27:14365-14374.

Hassabis D, Kumaran D, Vann SD, Maguire EA (2007b) Patients with hippocampal amnesia cannot imagine new experiences. Proc Natl Acad Sci U S A 104:1726-1731.

Jeneson A, Mauldin KN, Squire LR (2010) Intact working memory for relational information after medial temporal lobe damage. J Neurosci 30:13624-13629.

Kan IP, Giovanello KS, Schnyer DM, Makris N, Verfaellie M (2007) Role of the medial temporal lobes in relational memory: neuropsychological evidence from a cued recognition paradigm. Neuropsychologia 45:2589-2597.

Kirwan CB, Bayley PJ, Galván VV, Squire LR (2008) Detailed recollection of remote autobiographical memory after damage to the medial temporal lobe. Proc Natl Acad Sci U S A 105:2676-2680.

Klein SB, Loftus J, Kihlstrom J (2002) Memory and temporal experience: the effects of episodic memory loss on an amnesic patient's ability to remember the past and imagine the future. Soc Cogn 20:353-379.

Levine B, Svoboda E, Hay JF, Winocur G, Moscovitch M (2002) Aging and autobiographical memory: dissociating episodic from semantic retrieval. Psychol Aging 17:677-689.

Leyhe T, Müller S, Eschweiler GW, Saur R (2010) Deterioration of the memory for historic events in patients with mild cognitive impairment and early Alzheimer's disease. Neuropsychologia 48:4093-4101.

Luck D, Danion JM, Marrer C, Pham BT, Gounot D, Foucher J (2010) The right parahippocampal gyrus contributes to the formation and maintenance of bound information in working memory. Brain Cogn 72:255-263.

Maguire EA, Hassabis D (2011) Role of the hippocampus in imagination and future thinking. Proc Natl Acad Sci U S A 108:E39.

Maguire EA, Vargha-Khadem F, Hassabis D (2010) Imagining fictitious and future experiences: evidence from developmental amnesia. Neuropsychologia 48:3187-3192.

McKinlay A, McVittie C, Della Sala S (2010) Imaging the future: does a qualitative analysis add to the picture? J Neuropsychol 4:1-13.

Mitchell KJ, Johnson MK, Raye CL, D’Esposito M (2000) fMRI evidence of age-related hippocampal dysfunction in feature binding in working memory. Brain Res Cogn Brain Res 10:197-206.

Moulton ST, Kosslyn SM (2009) Imagining predictions: mental imagery as mental emulation. Philos Trans R Soc Lond B Biol Sci 364:1273-1280. 
Nadel L, Moscovitch M (1997) Memory consolidation, retrograde amnesia and the hippocampal complex. Curr Opin Neurobiol 7:217-227.

Nadel L, Samsonovich A, Ryan L, Moscovitch M (2000) Multiple trace theory of human memory: computational, neuroimaging, and neuropsychological results. Hippocampus 10:352-368.

Okuda J, Fujii T, Ohtake H, Tsukiura T, Tanji K, Suzuki K, Kawashima R, Fukuda H, Itoh M, Yamadori A (2003) Thinking of the future and past: the roles of the frontal pole and the medial temporal lobes. Neuroimage 19:1369-1380.

Olson IR, Page K, Moore KS, Chatterjee A, Verfaellie M (2006) Working memory for conjunctions relies on the medial temporal lobe. J Neurosci 26:4596-4601.

Piekema C, Kessels RP, Rijpkema M, Fernández G (2009) The hippocampus supports encoding of between-domain associations within working memory. Learn Mem 16:231-234.

Rosenbaum RS, Gilboa A, Levine B, Winocur G, Moscovitch M (2009) Amnesia as an impairment of detail generation and binding: evidence from personal, fictional, and semantic narratives in K.C. Neuropsychologia 47:2181-2187.

Schacter DL, Addis DR (2007) The cognitive neuroscience of constructive memory: remembering the past and imagining the future. Philos Trans $\mathrm{R}$ Soc Lond B Biol Sci 362:773-786.

Schacter DL, Addis DR (2009) On the nature of medial temporal lobe contributions to the constructive simulation of future events. Philos Trans R Soc Lond B Biol Sci 364:1245-1253.
Schacter DL, Addis DR, Buckner RL (2007) Remembering the past to imagine the future: the prospective brain. Nat Rev Neurosci 8:657-661.

Seidman LJ, Faraone SV, Goldstein JM, Kremen WS, Horton NJ, Makris N, Toomey R, Kennedy D, Caviness VS, Tsuang MT (2002) Left hippocampal volume as a vulnerability indicator for schizophrenia: a magnetic resonance imaging morphometric study of nonpsychotic first-degree relatives. Arch Gen Psychiatry 59:839-849.

Squire LR, Alvarez P (1995) Retrograde amnesia and memory consolidation: a neurobiological perspective. Curr Opin Neurobiol 5: $169-177$.

Squire LR, van der Horst AS, McDuff SG, Frascino JC, Hopkins RO, Mauldin KN (2010) Role of the hippocampus in remembering the past and imagining the future. Proc Natl Acad Sci U S A 107:19044-19048.

Steinvorth S, Levine B, Corkin S (2005) Medial temporal lobe structures are needed to re-experience remote autobiographical memories: evidence from H.M. and W.R. Neuropsychologia 43:479-496.

Suddendorf T, Corballis MC (2007) The evolution of foresight: what is mental time travel, and is it unique to humans? Behav Brain Sci 30:299 313; discussion 313-351.

Thaiss L, Petrides M (2008) Autobiographical memory of the recent past following frontal cortex or temporal lobe excisions. Eur J Neurosci $28: 829-840$.

Tulving E (1985) Memory and consciousness. Can Psychol 25:1-12.

Weiler JA, Suchan B, Daum I (2010) Foreseeing the future: occurrence probability of imagined future events modulates hippocampal activation. Hippocampus 20:685-690. 